\title{
Flight Guidance and Control System Modeling and Design Based on AADL
}

\author{
Wei Zhong ${ }^{1, a}$, Lichen Zhang ${ }^{2, b}$ \\ School of Guangdong University of Technology, Guangzhou 510000, China. \\ a1240500584@qq.com
}

Keywords: FG\&CS, AADL, FC, FG.

\begin{abstract}
Flight Guidance and Control System is widely used in the world.which effectively improve the efficiency of play operation ,reduce system maintenance and construction costs,etc. so,This paper propose the use of structural analysis and design language(AADL) for Flight Management (FM).First,it is described by Flight Guidance and Control System ,then the paper use OSATE Modeling the system.Through the modeling proved the model can be used in the Flight guidance and Control system.
\end{abstract}

\section{Introduction}

With the development of aviation industry, the design of aircraft control system has changed a lot ,More and More people attention to the automatic guidance and control system.

The flight guidance and control system (FGCS) of modern general transport plane include flight control system (FC), the flight management system (FM), automatic flight control system (AFC).It is also generally contains structure control (SC) and active load control (ALC). The FG\&CS consists of the following components[1]:

1、FC: Its task is executed by FCS[2].This system allows the pilot to use the control bar, rudder pedals and a series of control devices to manually fly a plane.Through feedback flight information and feedback algorithm, FCS can automatically change the parameters of the aircraft flight directly, and help to real time accurately and independently (decoupling) tracking the flight parameters.Aircraft control unit will ensure flight safety, there are dangers security mechanisms to prevent pilots flying. The accelerator and brake speed is used to control the plane and flight path.If the system errors or system failure, the cockpit control will connect control surface in the form of electronic control.

2、FM: Flight management system (FMS) [3]can predict and optimize the parameters of the flight plan on an aircraft's performance or related algorithm, realization of automatic throttle and using the automatic pilot and the flight plan.Pilots can through the multi-function control and display unit ( MCDU) loaded to real-time optimization of flight plan or a plan in advance.

3、FG: Its task executed by AFCS scheme.The AFCS scheme is composed of autopilot and automatic throttle.The driver can control unit in the mask (glare shields control unit) set the flight mode and setting speed, altitude, vertical height, direction. The AFCS scheme used in long distance of aircraft autopilot.

Architecture Analysis and Design Language is a language that use the advantages from UML and Marte while also be able to write in text and graphics[4].To describe the unfunctional quality of complicated embedded systems, such as real-time requirement, safety requirement , schedulablity.AADL offers a standard and accurate way so that the programmer could analyse the schedulablity and safety about components they used, ensure the consistency from the model to the codes they turned.Impeccable modeling, test and Verify tools could make the user making a model faster, produce the response code automatically, and testing at the same time.In this way, the system designer could make the components better and do some functional and quality estimates in the designing phase.So using AADL to model the CPS could describe its unfunctional quality well, 
guranteeing the high quality codes, it will be more and more important in designing and producing systems which combined information and physical functions, focus on running-time and safety.

\section{Design and Modeling of FG\&CS}

First of all, The flight guidance and control system need the overall modeling, it peripherals include sensors, radio receivers, control equipment, throttle control, screen control unit, route control unit, display, drive controller, flight control, emergency equipment, etc. Figure 1 represents the overall modeling.

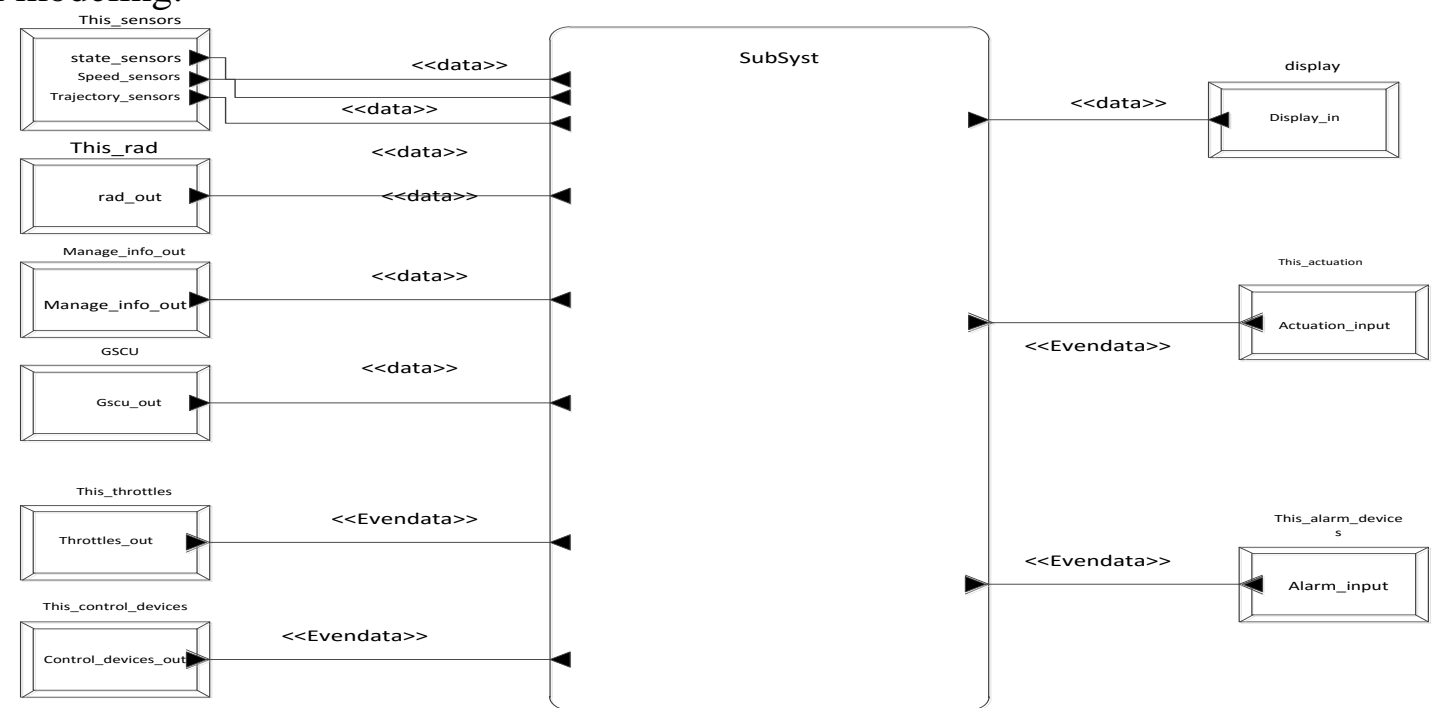

Fig.1 The overall modeling of FG\&CS

We design overall System part of the code is as follows:

system implementation complete.syst

subcomponents

this_sensors: device Sensors;

this_rad: device RAD;

this_manage_device: device manage_device;

SubSyst: system SubSys::subsystems.with_components;

connections

complete_conn0: port this_sensors.trajectory_sensors->SubSyst.trajectory_sensors_in;

bus10: bus access this_bus -> this_alarm_devices.bus_access;

end complete.syst;

flight guidance and control system provided four main functions: the flight management (FM), flight guidance(FG), the flight control (FC), and the structure and drive control (SLC).For navigation and control system for aircraft respectively set up four processes, modeling.Fig.2 show the model of them. 


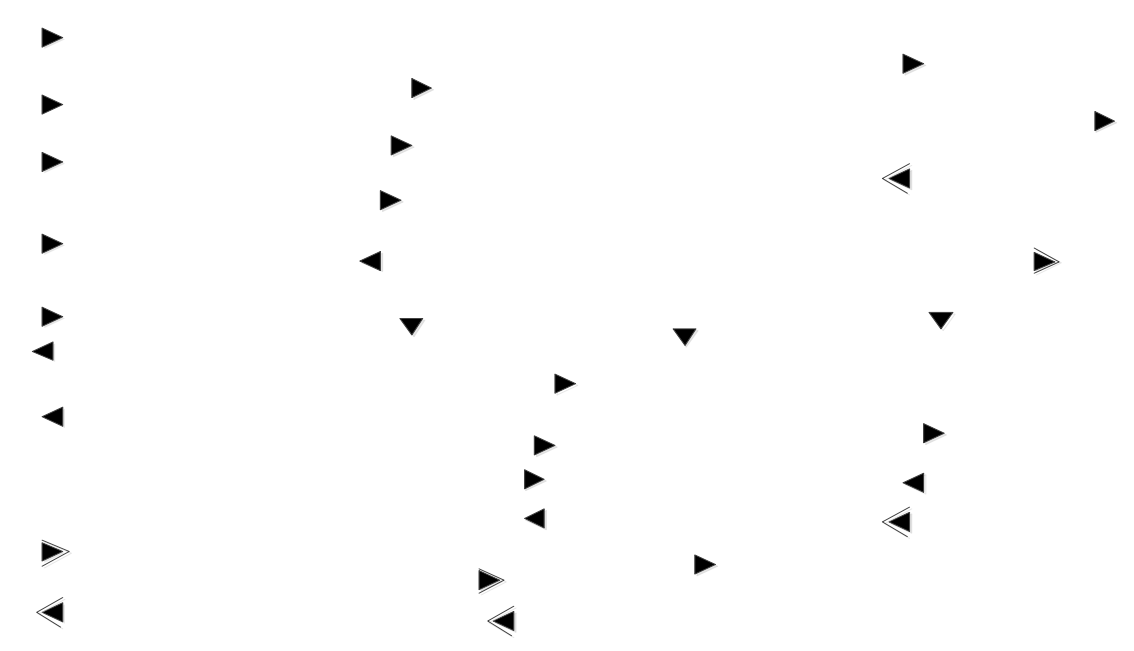

Fig.2 The process level modeling

We design process System part of the code is as follows:

system implementation subsystems.with_components

subcomponents

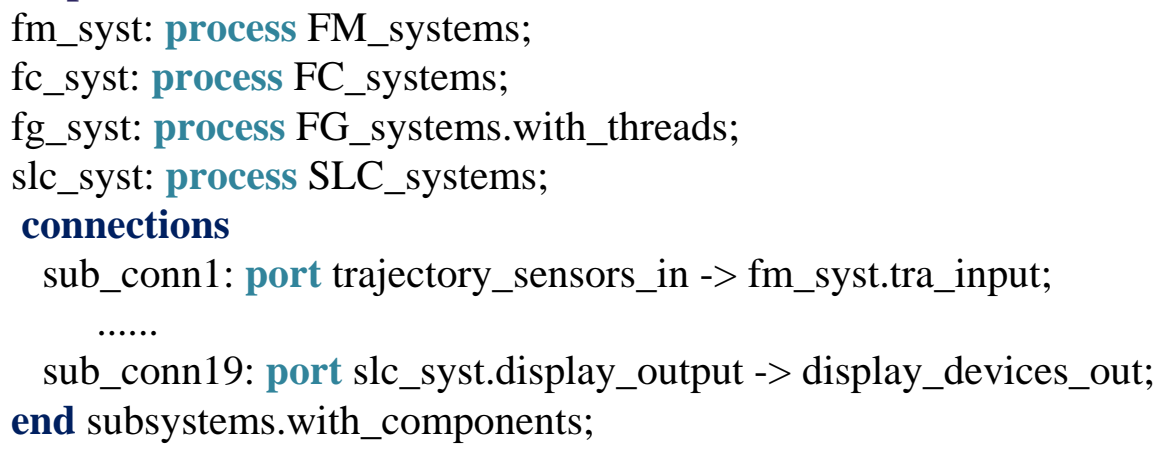

\section{Design and Modeling of Flight Guidance Service}

We take the plane down this scenario to analysis flight guidance service.The input data of the whole process are: flight management system management of the input data, sensor transmits the plane speed, path information, and the pilot in flight through the control panel, is balancing the plane down[5].The whole process of decline is a place where drivers test flight technology.At the same time the output is display devices and execution. flight guidance and control services can be divided into three threads, thread guide, speed and route tracking thread, and signal stability and thread. Fig.3 show the model of flight guidance service. 

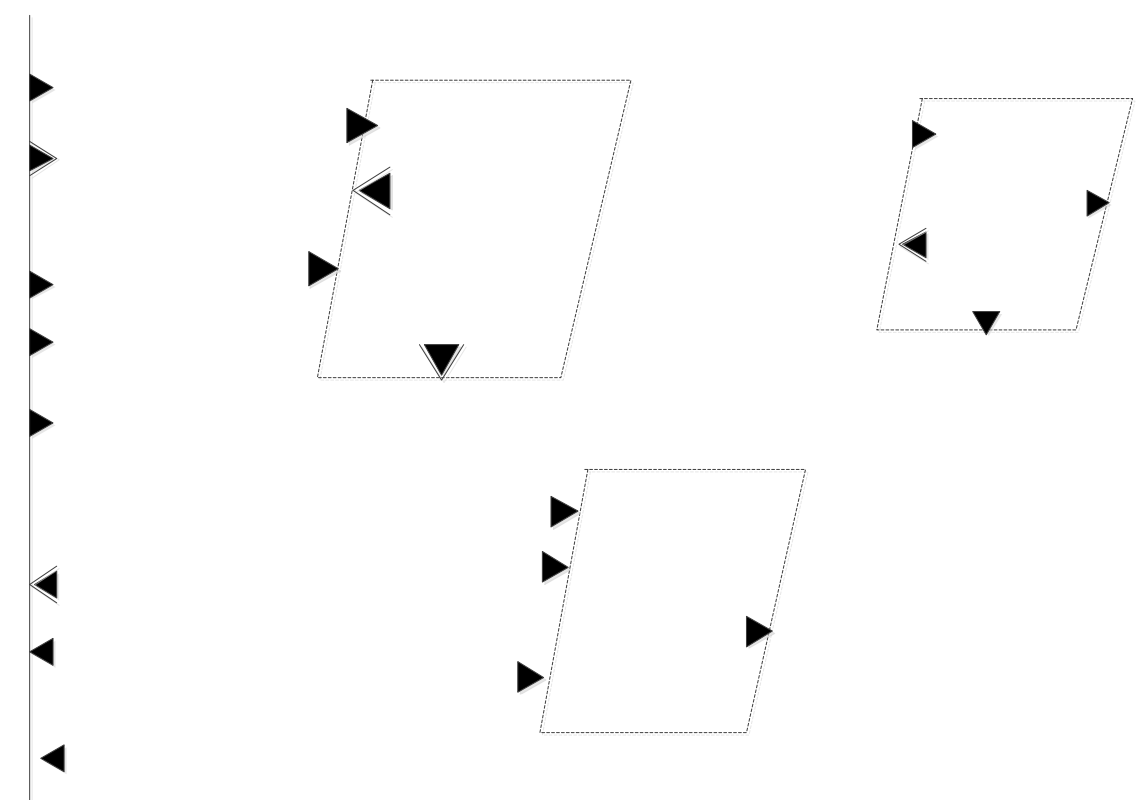

Fig.3 The thread level modeling

We design process System part of the code is as follows:

process implementation FG_systems.with_threads

\section{subcomponents}

this_guidance_law: thread guidance_law;

this_traking_law: thread traking_law;

this_augmentation_rules: thread augmentation_rules;

connections

fg_conn1: port fm_manage_input->this_guidance_law.manange_inbuf_port;

fg_conn11: port this_augmentation_rules.fc_outbuf_port->fg_output;

end FG_systems.with_threads;

\section{Design and Modeling of SLC}

Structural control and navigation system is simple, it mainly includes three threads: filter thread, control rules thread, and the amplified signal thread. Fig.4 show the model of SLC.

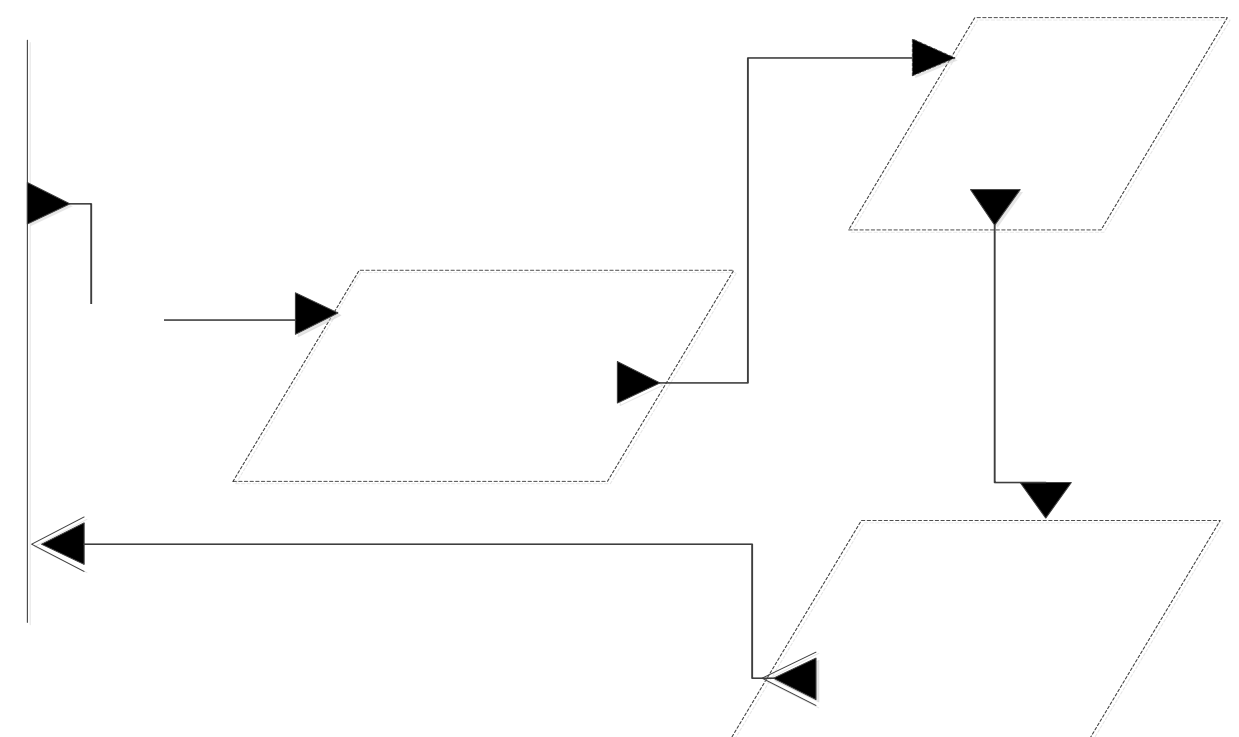

Fig.4 The model of SLC 


\section{Summary}

In this paper, we propose flight guidance and control system design method based on AADL,we split FG\&C system to FC,FG,FM, SLC systems and discusse,then made a reasonable model. Follow-up studies will further FG\&C system model based on AADL, the system can dispatch, safety and reliability analysis and verification methods.

\section{References}

[1]. JIM ESCH, Aviation Cyber-Physical Systems: Foundations for Future Aircraft and Air Transport, Proceedings of the IEEE , PROLOG,2013.101(8).

[2]. Lichen Zhang, Designing Big Data Driven Cyber Physical Systems Based on AADL, 2014 IEEE International Conference on Systems, Man, and Cybernetics, October 5-8, 2014, San Diego, CA, USA.

[3]. Peter H. Feiler, Bruce Lewis, The SAE Avionics Architecture Description Language (AADL) Standard: A Basis for Model-Based Architecture-Driven Embedded Systems Engineering, Software Engineering Institute, 2003.

[4]. Krishna Sampigethaya, Member IEEE , Radha Poovendran, Senior Member IEEE , Sudhakar Shetty, Terry Davis, Member IEEE,Chuck Royalty, Future E-Enabled Aircraft Communication sand Security: The Next 20 Years and Beyond, Proceedings of the IEEE(INVITED PAPE R),2011.99(11), 0018-9219

[5]. P. H. Feiler, D. P. Gluch, J. J. Hudak. The Architecture Analysis \&Design Language (AADL): An Introduction. CMU/SEI-2006-TN-001,Carnegie Mellon University \& Software Engineering Institute,2006.

[6]. GILLES, Olivier. HUGUES, Jérôme. Expressing and enforcing user-defined constraints of AADL models. In: Proceedings of the 5th UML and AADL Workshop (UML and AADL 2010) 